\title{
Experimental Challenges when Measuring Partial Discharges under Combined DC and High Frequency AC Voltage
}

\author{
Pål Keim Olsen, Ingrid Roen Velo and Frank Mauseth \\ Norwegian University of Science and Technology, \\ Department of Electrical Power Engeneering
}

\begin{abstract}
The present paper reviews some of the challenges involved in designing and building an experimental setup for measurement of partial discharge under combined AC and DC voltage. A design of a modular test cell is discussed, as well as all parameters relevant to proper control of the experiment conditions. The described experimental setup is capable of producing sinusoidal AC voltage at a variable frequency up to 5 $\mathrm{kHz}$ superimposed a high DC voltage. The challenges related to intermittent noise reduction was remedied by placing the setup in a Faraday cage and controlling the setup from the outside by optical cables. All equipment in the test setup was connected to the power grid through low-pass filters.
\end{abstract}

\section{Introduction}

The use of HVDC transmission systems have been steadily increasing since the first installation of the subsea link between mainland Sweden and the island of Gotland in 1954. HVDC transmission has found its niche in three main areas: extremely long distance transmission, connection between unsynchronized AC power systems and underwater transmission. The last decade HVDC systems have gained territory in power transmission to offshore wind parks and oil platforms, using IGBT-VSC technology in the HVDC converter stations. As AC to DC conversion will result in a high frequency ripple voltage on the DC voltage, it will also affect the partial discharge activity in HVDC insulation [1]. Good experimental practice is paramount to push the research within this field further, the present paper will focus on the experimental challenges when designing and building a setup to measure partial discharges (PD) under combined HVDC and high frequency AC voltage.

\section{Preliminary considerations}

Designing partial discharge detection experiments for DC or combined DC and AC voltage has some challenges, which is not widely reported in the literature. It is instructive to consider some of the differences of partial discharges under pure AC voltage and pure DC voltage. The following discussion is based on the $\mathrm{ABC}$ circuit model for cylindrical cavities in a dielectric material [1].

\subsection{Discharge repetition rate}

One of the most important differences between PD under $\mathrm{AC}$ voltage and $\mathrm{PD}$ under $\mathrm{DC}$ voltage is the repetition rate of the discharges, $n[1 / \mathrm{s}]$. The respective $\mathrm{AC}$ and DC voltage must be higher than the measured partial discharge inception voltage (PDIV), the PD repetition rate is then increasing when the applied voltage is increasing. The PDIV under DC can be difficult to define, but can be taken as the voltage at which the repetition rate is higher than some chosen level. The PDIV at AC (PDIVAC) is governed by the permittivity and geometry of the cavity, the PDIV for DC (PDIVDC) is governed by the conductivity and geometry of the cavity, so these values can differ, but usually not more than one order of magnitude. Under pure $\mathrm{AC}$, the repetition rate is proportional to the frequency of the applied sinusoidal $\mathrm{AC}$ voltage, $f_{A C}$ :

$$
n_{A C} \propto f_{A C} \cdot\left(\frac{\widehat{V}_{A C}}{V_{P D I V, A C}}\right)
$$

Under DC the repetition rate is inverse proportional to $\tau$, the time constant of the series coupled cavity and bulk impedance:

$$
n_{D C} \propto \frac{1}{\tau} \cdot\left(\frac{V_{D C}}{V_{P D I V, D C}}\right)
$$

For a cylindrical cavity:

$$
\frac{1}{\tau}=\frac{\sigma_{c} D+\sigma_{b}}{\varepsilon_{c} D+\varepsilon_{b}}
$$

where

$$
D=\frac{H-h}{h}
$$

and $H$ is the total thickness of the sample, $h$ is the height of the cavity, see Fig. 1. For normal values of conductivity and permittivity of insulation materials and air, $1 / \tau$ is 4 to 5 orders lower than $f_{A C}$. This means there are a much less discharges produced under a DC voltage, in the range of 1 to 1000 discharges per minute. 


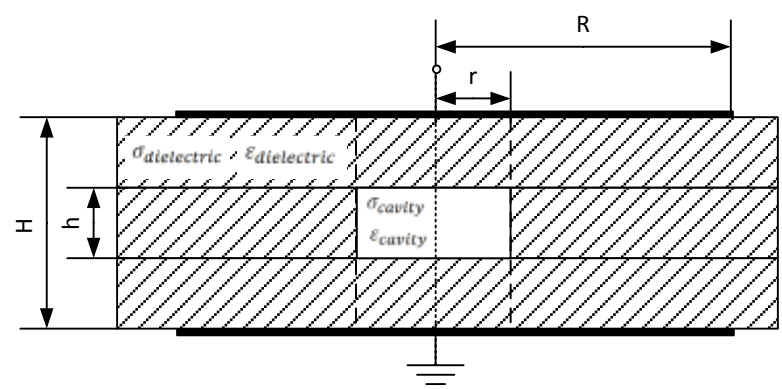

Fig. 1 Structure of test object, three layer of films with cylindrical hole in the middle layer

When an $\mathrm{AC}$ voltage is overlaid the $\mathrm{DC}$ voltage, the repetition rate will increase according to:

$$
n_{D C+A C} \propto \frac{1}{\tau} \cdot\left(\frac{V_{D C}}{V_{P D I V, D C}}\right) \cdot\left(\frac{1}{1-\frac{\widehat{V}_{A C}}{V_{P D I V, A C}}}\right)
$$

When the AC voltage is close to the PDIVAC, the repetition rate will increase rapidly. The repetition rate is given by (1), i.e. many orders higher. When doing experiments it is important to consider if $\widehat{V}_{A C} \geq$ $V_{P D I V, A C}$ due to the radical increase in repetition rate. In this paper it assumed that $\widehat{V}_{A C}<80 \% \cdot V_{P D I V, A C}$.

The repetition rate given by (5) is directly proportional to the conductivity of the cavity and the bulk material, and inversely proportional to the permittivity, according to (3). The conductivity of the materials is sensitive to temperature, amplitude of the electric field and the ion species present due to moisture and contaminants. The permittivity, however, can be considered constant for the same conditions. The conductivity is also time dependent, the measured current exhibit the following tendency for PET [2] and PE [3]:

$$
I=A \cdot t^{-n}
$$

where $A$ and $n$ depend on bulk material, electrode material, electric field magnitude and temperature.

\subsection{Polarity of discharges and waiting time for start electrons}

In contrast to $\mathrm{AC}$, the discharges under $\mathrm{DC}$ voltage are unipolar as the field direction does not alternate. This influence the supply of starting electrons: any electrons must be supplied by bulk conduction [4]. At AC, starting electrons may be available from previous discharges on the cavity surface. The time lag for available starting electrons is therefore expected to be different for $\mathrm{AC}$ and DC. The combination of time lag and rise time of voltage influence the overvoltage before discharge. Increased overvoltage will increase the discharge magnitude and govern the discharge mechanism [5].

\subsection{Discharge magnitude}

In [4] it was found that the voltage dropped just a couple of volts during a discharge under DC voltage, thus the residual voltage was very close to the cavity ignition voltage. A high residual voltage will make the discharge magnitude smaller, and increase the frequency of the discharges.

\subsection{Space charges and electrostatic forces on the cavity walls}

When the DC voltage is above some threshold, the field distribution inside the dielectric can be highly inhomogeneous due to space charge accumulation. For XLPE this threshold is $10-20 \mathrm{kV} / \mathrm{mm}$ [6]. Space charges increases the local field, and complicates the calculation of field distribution in the bulk and the cavity. It may also influence the electrostatic forces on the cavity walls. In the literature, the diameter of the artificial cavities can be as large as $10 \mathrm{~mm}$ and with a film thickness down to $50 \mu \mathrm{m}$. Samples with these dimensions are vulnerable to deformation of the cavity at high temperatures [7], with potential to significantly influence the partial discharge characteristics of the cavity.

\subsection{The combined effect}

The net result of the effects mentioned above is that PD under DC voltage:

- Have smaller magnitude

- Occurs less frequently

- Is unipolar

- Is dominated by the conductivity of the insulation, which is highly influenced by parametres like time, temperature, electric field stress, electrode material and contaminants

- Can be influenced by electrostatic forces

- Have more complex field distribution above threshold for space charge accumulation

\section{Design of experiment}

\subsection{Requirements}

From the discussion in the previous section, it is clear that an experiment for measuring PD under combined DC and AC voltage must fulfill the following requirements:

- $\quad$ Electromagnetic noise immunity must be very high

- The temperature must be controlled accurately 
- The mechanical pressure on the sample must be high, to avoid discharges between film layers

- Careful sample preparation, to avoid contamination and to increase reproducibility of experiments

\subsection{Choice of sample type and cavity}

It is preferable to choose a material that is used in electrical apparatus, and where there is substantial scientific literature on the conductivity and permittivity of the material. A layered sample with a cylindrical cavity (Fig. 1) facilitates an easy and repeatable preparation of samples. For such reasons, a biaxially oriented PET film was chosen. The film can be supplied in many different thicknesses, from 50 to $350 \mu \mathrm{m}$. The thickness of the sample and the height of the cavity can then easily be changed.

The cavity size choice was influenced by two aspects:

- The high voltages which are necessary to generate partial discharge at DC cause an increased probability of occurrence of discharges outside the sample, thus a thin film is preferable. This reduces the DC voltage needed to start discharges in the sample.

- The cavity diameter should not be too small due to inhomogeneity of the field inside the cavity and not too large due to the danger of deformation of the cavity [8].

For the experiments a cavity with $2 \mathrm{~mm}$ in diameter was chosen, the film thickness was $100 \mu \mathrm{m}$.

\subsection{Test cell}

The test cell is shown in Fig. 2, it is modular and can easily be taken apart. The design of a subcomponent can be changed without doing changes to other subcomponents. These aspects facilitate improvement of the design, and makes the test cell very flexible. Possible versions of the bottom electrode ranges from guarded (for conductivity measurements), unguarded (for PD detection) and transparent (for optical detection). The test cell in this paper use an unguarded brass bottom electrode, and a brass top electrode with Rogowski profiles. Mechanical pressure is controlled by weights on the top electrode, temperature is controlled by switching the resistive heat coil, oil is used as both thermal conductor and for suppressing discharges at the edges of the electrodes. O-rings was used to get the cell oil-, vacuum- and airtight. PT 100 elements measure the temperature inside the bottom brass electrode, and in the oil.

\subsection{Noise suppression}

The following measures was taken to reduce constant and intermittent noise:

- Complete setup placed inside faraday cage, all power provided through filtered mains.

- Control and data over optical cables, to avoid any antenna effects

- Star point ground, focus on avoiding ground loops

- Clean lab environment

- $\quad$ Ferrite cores on all USB and signal leads in and out of control box

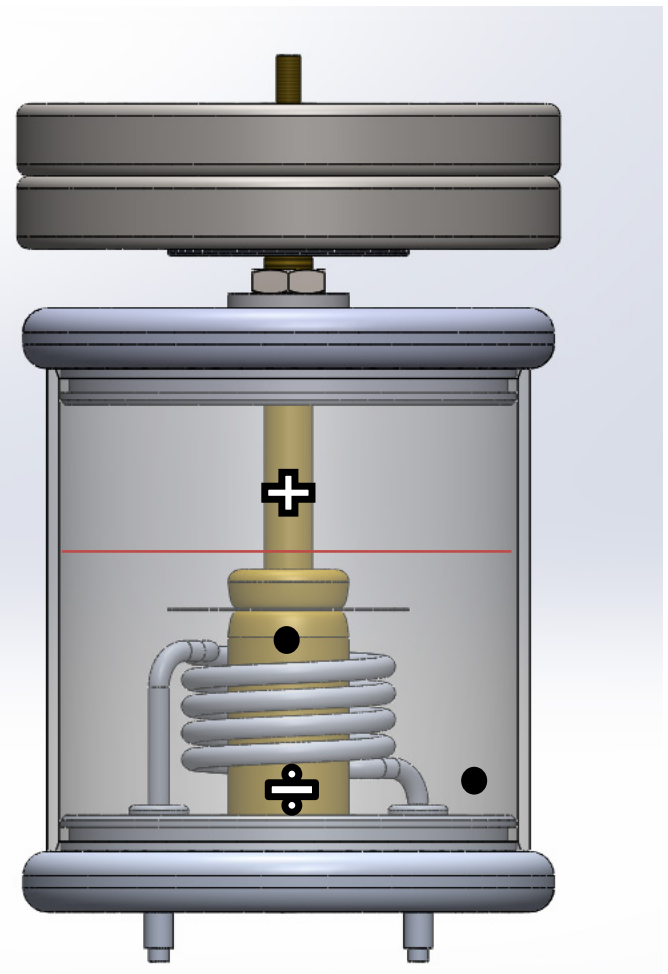

Fig. 2 Test cell. Resistive coil element (grey spiral) heats oil and sample, oil level marked by red line. Brass electrodes in

beige, + sign marks top electrode, $\div$ sign marks bottom electrode. The temperature sensors are marked by black dots. Mechanical pressure on sample controlled by weights shown at the top.

\subsection{Generating circuit}

The high frequency AC voltage is generated by a high voltage oscillator and stepped up using a voltage transformer $(110 \mathrm{~V}: 11 \mathrm{kV})$. A buffer capacitance is connected in series to block any DC current from entering the transformer's secondary side, the capacitance is high compared to the coupling capacitor and the test object, so that most of the $\mathrm{AC}$ voltage drop is over the test object. The high inductance of the voltage transformer makes the AC generation branch a high impedance path for the discharge current. A highly stabilized HVDC source supply the DC voltage through a buffer resistor. The buffer resistor blocks the AC current injection into the DC source, attenuate noise 
coming from the DC source and makes the DC branch a high impedance path for the discharge currents.

\subsection{Measuring circuit}

The measurement circuit is the standard straight circuit for PD detection. A coupling capacitor, $C_{k}$, provide a low impedance path for detection of the discharge currents. The resistor $R_{k}$ provides a way for automatically discharging the capacitances after a measurement. $R_{k}$ and $C_{k}$ also makes it possible to measure the combined voltage over the test object. A measuring impedance consisting of a HF current transformer in series with a $30 \mu \mathrm{F}$ capacitance is placed in series with the coupling capacitor and the test object, each measuring impedance is connected to an Omicron MPD 600 amplifier and digitizer. The gain level is set as high as possible at the MPD in the test object branch, detecting the discharges with magnitude from $2 \mathrm{pC}$ to $100 \mathrm{pC}$, while the discharges larger than $100 \mathrm{pC}$, up to $750 \mathrm{pC}$, are picked up by the MPD in the coupling capacitor branch. The MPD at the coupling capacitor has a lower gain and can detect larger discharges before saturation. In a previous setup [1], the MPDs was connected to the same measuring impedance, but this effectively halved the signal-to-noise ratio, making it difficult to detect the polarity of the small discharges. The present setup allow a better signal-to-noise ratio and preserve the polarity information.

\subsection{Remote control of setup}

All data and control signals was transferred via optical cables. A NI USB 6216 was used to measure temperature, control heat coil and voltage output of the $\mathrm{AC}$ and DC source. Frequency was controlled manually, by using the 10 turn potmeter on the AC source. The voltage sequence output and temperature controller was implemented using Labview.

\section{Test procedure}

A systematic approach is vital to acquire scientific confidence in the final test results. The test setup is comprised of many sub-systems; the DC and AC generating branch, PD measurement system, temperature control, voltage control, test cell etc. The lab environment will also influence the setup. Here, a test procedure is outlined which intends to reduce the test complexity, and work its way from component level to full assembly of the test setup:

1. Sub-system test: verification of function and noise level of separate components

2. System test: verification of function of assembled components and noise level

3. Main test: PD detection in the PET test objects

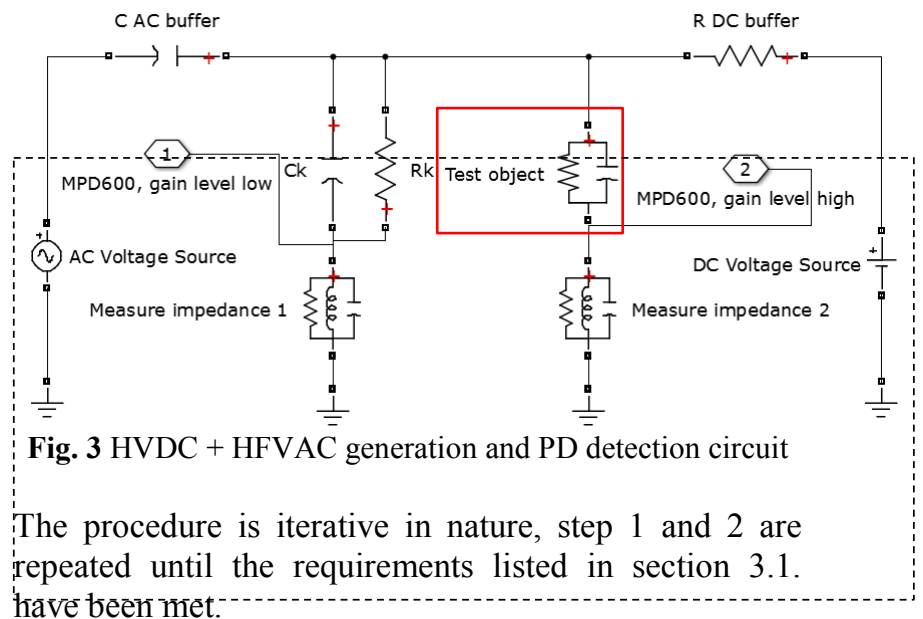

have been met.

In step 1 and 2 the following tools was used:

- PD calibrator at $2 \mathrm{pC}$ to check successful polarity hit rate

- High bandwidth oscilloscope measurements of voltage waveforms

- Check FFT up to $30 \mathrm{MHz}$, to identify frequency band with lowest noise levels

- 24 hour PD measurement to check for intermittent noise

- 24 hour temperature measurement

- 24 hour voltage stability measurement

In step 3, the following procedure was followed.

1. 24 hour PD measurement on solid PTFE sample at the highest $\mathrm{DC}$ and $\mathrm{AC}$ voltage planned for the final experiments. Temperature $60{ }^{\circ} \mathrm{C}$.

2. 24 hour PD measurement on 3 sheets of PET, without cavity, highest DC and AC voltage. Temperature $60{ }^{\circ} \mathrm{C}$. Various mechanical pressure.

3. Measurements on samples with cylindrical cavity.

\section{Observations from system tests}

\subsection{High frequency effects}

Adding a high frequency component to the DC voltage also add noise to the setup. Noise detection must be assessed for each AC voltage frequency and careful selection of the detection frequency band is necessary to cope with the higher level of disturbance. The circuit elements changes when increasing the frequency, especially the impedance and voltage ratio of the voltage transformer. A resonance was observed around $4 \mathrm{kHz}$. The total harmonic distortion was below $1 \%$ for 50-5000 Hz and a voltage between 1-2 kVrms.

The maximum voltage at high frequency is limited by: 

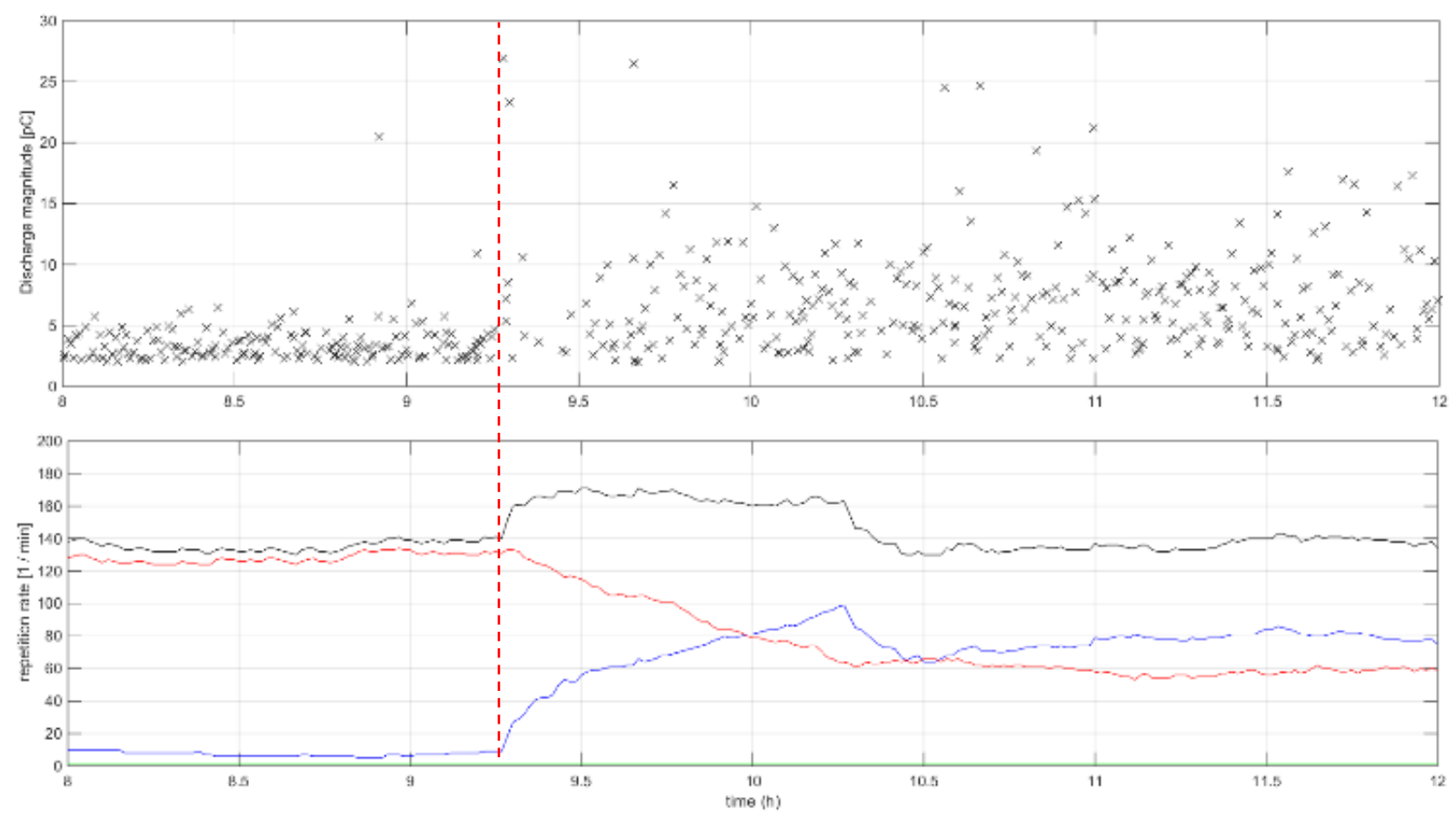

Fig. 4 Discharges at $10 \mathrm{kVDC}+500 \mathrm{Vrms}$ at $500 \mathrm{~Hz}, 2 \mathrm{~kg} / \mathrm{cm}^{2}$ pressure, $60{ }^{\circ} \mathrm{C}$. AC voltage ramped up at time marked by red dashed line. Upper plot: PD event and magnitude. Lower plot: Repetition rate - black line: all discharges; red line: discharges above $6 \mathrm{pC}$; blue line: discharges under $6 \mathrm{pC}$

- The capacitive load

- $\quad$ Power rating of the transformer.

- The voltage ratio at high frequency

The transformer in this setup has a rating of $30 \mathrm{VA}$, limiting the continuous voltage at $5 \mathrm{kHz}$ to $1 \mathrm{kVrms}$. To allow for higher voltage at high frequency both the coupling capacitance and the test object capacitance must be lowered; the ratio between the two capacitances must be kept the same, to preserve high accuracy in the PD measurements.

The phase position of PDs is important information for the experiments. The Omicron MPD 600 have an AC frequency limitation of $2.1 \mathrm{kHz}$, for other commercial detection devices the limit is $400 \mathrm{~Hz}$. For frequencies above $50 \mathrm{~Hz}$ the accuracy of the PD phase position deteriorates, and from $2 \mathrm{kHz}$ the phase position of the $\mathrm{PD}$ is lost completely. This issue have not been resolved.

\subsection{Noise from instrument power supplies and control box}

Accidentally, switch mode power supplies was employed to provide $5 \mathrm{~V}$ and $12 \mathrm{~V}$ to the control and measurement instruments. These power supplies was replaced with old transformer-full bridge rectifier types, due to the conducted and wave transmitted noise from the switch mode supplies. Experiments revealed that noise levels were very dependent on the position of the control box, due to wave transmitted noise. The control box was put in a shielded container and ferrite cores damped common mode noise coming from the leads in and out of the box.

\subsection{PD detection frequency band}

Initially, a narrow bandwidth of $9 \mathrm{kHz}$ was used, making it easier to find a low noise frequency band, but this hindered the consistent polarity detection. Increasing the bandwidth to $300 \mathrm{kHz}$ increased the polarity hit rate to $99 / 100$. The optimum center frequency for this setup was $200 \mathrm{kHz}$.

\section{Results from the main experiment}

\subsection{Samples with cavity, $r=1 \mathrm{~mm}$}

Measurement shown in Fig. 4, $10 \mathrm{kVDC}$ applied at $60{ }^{\circ} \mathrm{C}, \mathrm{AC}$ voltage ramped up at $10 \mathrm{~V} / \mathrm{s}$ after 8 hours, when the discharge repetition rate had stabilized.

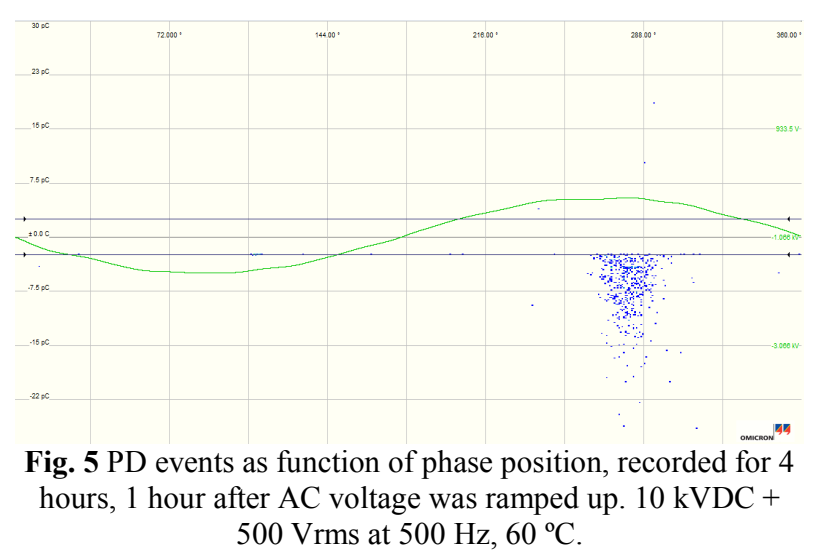




\subsection{Samples without cavity}

For a pressure of $2 \mathrm{~kg} / \mathrm{cm}^{2}$, the number of discharges was negligible, between 15 to 75 discharges in 24 hours. For lower pressure, $1 \mathrm{~kg} / \mathrm{cm}^{2}$, the number of discharges increased by 1 to 2 orders.

\section{Discussion}

Parasitic discharges from the PET sheet interface layers was controlled to a certain extent, so that the number of discharges without cavity is much lower than with a cavity. At higher AC voltages than $500 \mathrm{~V}$, and lower pressures than $2 \mathrm{~kg} / \mathrm{cm}^{2}$, there is considerably more discharges. The lack of pressure outside the electrode edge may allow small pockets of air in the interface layer close to the electrode edges, where there is no mechanical pressure. Higher field strength is needed in PD detection under DC voltage, this increase the probability of discharge in this area. The high voltage electrode should be casted in epoxy to ensure mechanical pressure at the sample edges.

Visual inspection of the samples after measurements revealed oil ingress into several of the samples, even when the procedure for gluing at the edges was followed thoroughly. These measurements had to be discarded. A dry setup, with the oil level below the sample, would prevent this problem, and decrease the time and complexity in making the sample. The sample preparation and conditioning can also influence the results significantly, due to the effect of contaminants and humidity on the conductivity. The sample may also take up moisture from the oil during measurements. The water content in XLPE influence the conductivity by a factor 2-3.5 from dry to saturated sample [9], the moisture absorption at $23{ }^{\circ} \mathrm{C}$ for PET is $0.5 \mathrm{wt} \%$ several orders higher than XLPE. Better control of moisture would probably increase the reproducibility of the measurements.

As it have been indicated previously [1], the repetition rate of the larger discharges increase, the overall repetition rate seems less affected. This can be attributed to the time lag and changes in the overvoltage when AC voltage is added. If it is assumed that the waiting time for electrons is the same as before adding the ripple, the increased voltage rise rate of the ripple waveform will result in larger discharges, due to the increased probability of higher overvoltages. The PD events will center around the peak of the AC voltage as long as the rise rate of the DC voltage is much slower than the rise rate of the AC voltage, see Fig. 5.

\section{Conclusion}

The measurement of PD under combined DC and AC voltage is very demanding, due to the low discharge magnitude and repetition rate under DC voltage, and the requirement for correct polarity detection. Application of a high frequency AC voltage added noise to a setup which had very high noise suppression requirements. The phase position detection of the PD events was lost at frequencies higher than $2 \mathrm{kHz}$, due to limitations in the measurement device. Careful preparation of samples is necessary to achieve reproducibility of results. Care must be taken to avoid deformation of the cavity by electrostatic forces at high temperature and electric field strength.

Adding a ripple increased the repetition rate of larger discharges, this was attributed to the increase in overvoltage possible with faster voltage rise rate.

\section{References}

[1] P. K. Olsen, F. Mauseth, and E. Ildstad, "The effect of DC superimposed AC voltage on partial discharges in dielectric bounded cavities," in High Voltage Engineering and Application (ICHVE), 2014 International Conference on, 2014, pp. 1-4.

[2] D. M. Taylor and T. J. Lewis, "Electrical conduction in polyethylene terephthalate and polyethylene films," Journal of Physics D: Applied Physics, vol. 4, p. 1346, 1971.

[3] E. J. M. R. Bartnikas, Ed., Corona measurement and interpretation (Engineering Dielectrics. American society for testing and materials, 1979, p.^pp. Pages.

[4] U. Fromm, "Partial Discharge and Breakdown Testing at High DC Voltage," PhD Monograph, Delft University of Technology, 1995.

[5] J. C. Devins, "The 1984 J. B. Whitehead Memorial Lecture the Physics of Partial Discharges in Solid Dielectrics," Electrical Insulation, IEEE Transactions on, vol. EI-19, pp. 475-495, 1984

[6] G. Mazzanti, G. C. Montanari, and F. Palmieri, "Quantities extracted from space-charge measurements as markers for insulation aging," Dielectrics and Electrical Insulation, IEEE Transactions on, vol. 10, pp. 198-203, 2003.

[7] S. Beg and B. Salvage, "Discharge repetition in an air-filled cavity in polythene under high direct electric stresses at elevated temperatures," Electronics Letters, vol. 5, pp. 118-120, 1969.

[8] F. H. Kreuger, "Determination of the Internal Discharge Resistance of Dielectric Materials," Electrical Insulation, IEEE Transactions on, vol. EI-3, pp. 106-114, 1968.

T. A. Ve, F. Mauseth, and E. Ildstad, "Effect of water content on the conductivity of XLPE insulation," in Electrical Insulation and Dielectric Phenomena (CEIDP), 2012 Annual Report Conference on, 2012, pp. 649-653. 